\title{
Operational Transconductance Amplifier-Based Nonlinear Function Syntheses
}

\author{
EDGAR SÁNCHEZ-SINENCIO, SENIOR MEMBER, IEEE, JAIME RAMÍREZ-ANGULO, MEMBER, IEEE, \\ BERNABÉ LINARES-BARRANCO, AND ANGEL RODRÍGUEZ-VÁZQUEZ, MEMBER, IEEE
}

\begin{abstract}
We show that the operational transconductance amplifier (OTA), as the active element in basic building blocks, can be efficiently used for programmable nonlinear continuous-time function synthesis. Two efficient nonlinear function synthesis approaches are presented. The first approach is a rational approximation and the second is a piecewise-linear approach. Test circuits have been fabricated using a $3-\mu \mathrm{m}$ p-well CMOS process. The flexibility of the designed and tested circuits was confirmed.
\end{abstract}

\section{INTRODUCTION}

L ATELY, several authors [1]-[5] have been successfully using the operational transconductance amplifier (OTA) as the main active element in continuous-time active filters. The OTA is a programmable device ${ }^{1}$ and has only a single high-impedance node, in contrast to conventional op amps. This makes the OTA an excellent device candidate for high-frequency and voltage (or current) programmable analog basic building blocks.

The applicability of OTA's as components in the design of linear networks has been extensively discussed elsewhere [1], [6] and will not be repeated here.

The objective of this paper is to examine the applicability of OTA's as the basic elements in the design of nonlinear networks. There is not much reported in the literature on the use of OTA's for designing nonlinear components [7], [8]. Excellent contributions [9]-[11], [16] are reported of nonlinear circuits dealing with particular important nonlinear problems. In this paper, rather than try to tackle a specific problem, we focus our attention on a general approach dealing with nonlinear basic building blocks using OTA's as the main active elements. Nothing special was done to optimize the circuit performance but rather to explore the potential and applicability of the OTA-based nonlinear system approach.

Manuscript received February 4, 1989; revised August 8, 1989

E. Sánchez-Sinencio and J. Ramirez-Angulo are with the Department of Electrical Engineering, Texas A\&M University, College Station, TX $77843-3128$

B. Linares-Barranco is with the Department of Electrical Engineering, Texas A\&M University, College Station, TX 77843 and with the Departamento de Electrónica y Electromagnetismo, Universidad de Sevilla, 41012 Sevilla, Spain.

A. Rodríguez-Vázquez is with the Departamento de Electrónica y Electromagnetismo, Universidad de Sevilla, 41012 Sevilla, Spain.

IEEE Log Number 8931198.

${ }^{1}$ The output current $I_{0}$ of an OTA due to a differential input $v_{i d}$ is $I_{0}=g_{m} v_{i d}$, and $g_{m}$ is a voltage (current) controllable parameter [1], [6], $[7]$
We will present a number of nonlinear OTA circuits and will discuss two nonlinear analog function synthesis techniques based on these OTA basic building block elements. One synthesis approach uses rational approximation functions and the other uses a piecewise-linear approximation. Actual circuit implementations will be presented as well as the experimental results from several $3-\mu \mathrm{m}$ p-well CMOS test prototypes.

\section{BASIC BUILdING BLocks}

In this section we introduce the OTA-based fundamental nonlinear building blocks involved for the synthesis procedures.

\section{A. Multiplier Block}

A two-input four-quadrant multiplier has an output current given by

$$
I_{0}=K_{M} V_{1} V_{2}
$$

where the multiplier constant $K_{M}$ has units of amperes per square volt. If $V_{1}$ and $V_{2}$ can take any positive or negative sign, the multiplier is called a four-quadrant multiplier. This multiplier is represented in Fig. 1(a). The corresponding OTA-based implementations are shown in Fig. 1(b) and (c). The triangular block labeled $a$ represents a signal attenuator (with an attenuation factor $a$ ); its function is to equalize the maximum voltage swing of $V_{1}$ and $V_{2} .-V_{\text {BIAS }}$ is the usual bias control of the OTA. An active attenuator can be implemented in CMOS technology [15]. The signal level in the multiplier is restricted by a few hundred millivolts for $V_{I_{\mathrm{L}}}$ and $V_{I^{2}}$. Although not indicated in Fig. 1, assume the power supplies of the OTA's are $V_{D D}$ and $-V_{S S}$. The two options of Fig. 1(b) and (c) allow us to change the sign of $K_{M}$. Thus for the circuit of Fig. 1(b) we obtain

$$
I_{o_{1}}=g_{m_{1}} V_{1}=K\left(V_{I_{1}}+V_{S S T}\right) V_{1}
$$

and

$$
I_{o_{2}}=-g_{m_{2}} V_{1}=-K\left(V_{I_{2}}+V_{S S T}\right) V_{1}
$$

where $K$ is a process- and geometry-dependent constant, 


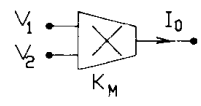

(a)

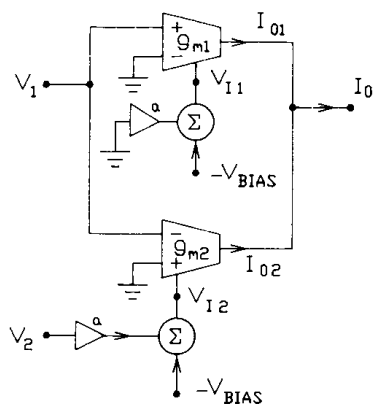

(b)

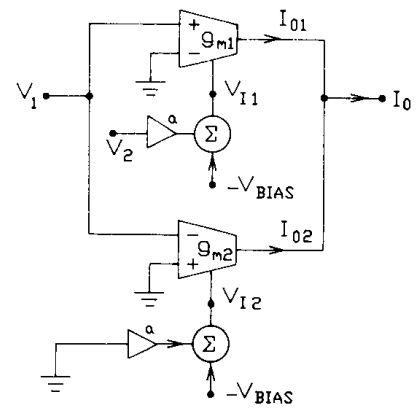

(c)

Fig. 1. Multiplier: (a) symbol, (b) OTA implementation 1, and (c) OTA implementation $2,0<a<1$

$V_{S S T}=V_{S S}-V_{t}$, and $V_{t}$ is a transistor threshold voltage ${ }^{2}$ :

$$
\begin{aligned}
& V_{I_{1}}=a 0-V_{\mathrm{BIAS}} \\
& V_{I_{2}}=a V_{2}-V_{\mathrm{BIAS}} .
\end{aligned}
$$

The output current $I_{0}$ becomes

$$
\begin{aligned}
I_{0}=I_{o_{1}}+I_{o_{2}}= & {\left[K\left(-V_{\mathrm{BIAS}}+V_{S S T}\right)\right.} \\
& \left.-K\left(a V_{2}-V_{\mathrm{BIAS}}+V_{S S T}\right)\right] V_{1} \\
I_{0}=-a K V_{1} V_{2}= & K_{M} V_{1} V_{2}, \quad K_{M}=-a K .
\end{aligned}
$$

A similar analysis of the circuit of Fig. 1(c) yields

$$
I_{0}=a K V_{1} V_{2}=K_{M} V_{1} V_{2}, \quad K_{M}=a K .
$$

Therefore, we can make the sign of $K_{M}$ positive or negative.

\section{B. Divider Block}

A two-input divider has an output which is the ratio of the two inputs, multiplied by a constant $K_{R}$ with dimensions in volts:

$$
V_{0}=K_{R} \frac{V_{1}}{V_{2}}
$$

A symbol of the divider is shown in Fig. 2(a), where $n$ and

${ }^{2} \mathrm{We}$ have assumed equal $K$ 's and threshold voltages $V_{t}$ 's for the OTA's
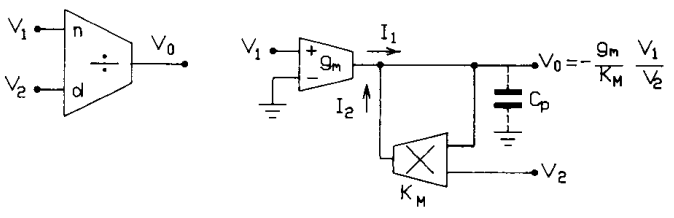

(a)

(b)

Fig. 2. Divider: (a) symbol and (b) OTA implementation.

$d$ stand for numerator and denominator, respectively. The corresponding circuit implementation using the multiplier symbol is shown in Fig. 2(b). Analysis yields

$$
\begin{aligned}
& I_{1}=g_{m} V_{1} \\
& I_{2}=K_{M} V_{0} V_{2} .
\end{aligned}
$$

By Kirchhoff's current law (KCL) we obtain

$$
I_{1}+I_{2}=g_{m} V_{1}+K_{M} V_{0} V_{2}=0 .
$$

Thus, the resulting output signal is proportional to the ratio of the input signals

$$
V_{0}=-\frac{g_{m}}{K_{M}} \frac{V_{1}}{V_{2}}=K_{R} \frac{V_{1}}{V_{2}} .
$$

Observe that $K_{R}$ can also be either negative or positive and $V_{2}=0$ is not allowed to avoid output saturation $\left(V_{o}\right)$. A closer look at the circuit of Fig. 2(b) is required to study stability. Assuming the dominant dynamic element is a parasitic capacitance $C_{p}$ at the output, (9a) is modified as

$$
I_{1}+I_{2}=s C_{p} V_{0}
$$

which yields

$$
V_{0}=\frac{-g_{m} V_{1}}{K_{M} V_{2}-s C_{p}} .
$$

Thus, $V_{0}$ has a pole located at

$$
s_{p}=\frac{K_{M}}{C_{p}} V_{2}
$$

Hence, in order for the circuit to be stable, its pole must be in the left-hand plane (LHP), which dictates that ${ }^{3}$

$$
K_{M} V_{2}<0 \text {. }
$$

A summary of the stability conditions are pictorially indicated by the hyperbolas of Fig. 3.

\section{Squaring and High-Powers (Exponentiation) Blocks}

A one-input squarer has an output proportional to the square of the input:

$$
I_{0}=K_{M} V_{i}^{2}
$$

${ }^{3}$ Observe that we are applying linear treatment to a nonlinear circuit This linear analysis is valid if for a certain instant $t_{x}, V_{1}\left(t_{x}\right)$ and $V_{2}\left(t_{x}\right)$ are considered constant; then $V_{o}$ will eventually reach its solution independent of the initial conditions. 


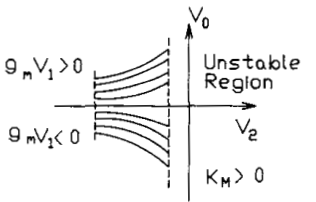

(a)

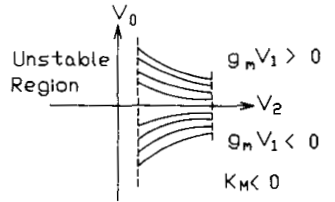

(b)

Fig. 3. Stability regions of divider. (a) Stable for $K_{M}>0$ and $V_{2}<0$. (b) Stable for $K_{M}<0$ and $V_{2}>0$.

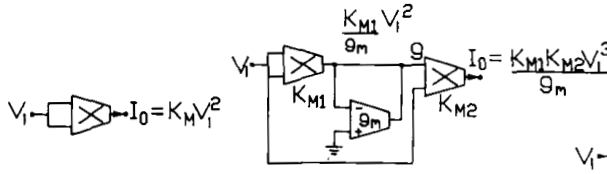

(a)

(b)

(c)

Fig. 4. Exponentiation (raising to a power) operation: (a) squarer, (b) cubic, and (c) $p$ th.

$v_{1}$

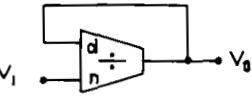

(a)

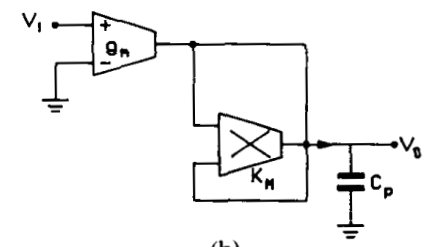

(b)
Fig. 5. Square rooter: (a) implementation and (b) OTA implementation.

The implementation of the squarer is obtained by simply using a multiplier with equal inputs, as shown in Fig. 4(a).

To obtain an exponentiation (raising to a power) circuit with an input $V_{i}$ and an output to be proportional to $V_{i}^{p}$, where $p$ is an integer greater than 2 , we require $(p+1) / 2$ multipliers for $p$ odd and $p / 2$ multipliers for $p$ even. Furthermore, since the proposed multipliers are of the transconductance type, the outputs must be converted into voltages for use as the inputs to subsequent multipliers. This can be done by connecting an equivalent resistor at the output. An equivalent resistor using an OTA [5], [6] is implemented by connecting the output to the negative OTA input and grounding the positive OTA input. An example for $p=3$ is shown in Fig. 4(b). It should be evident that a similar procedure can be followed to obtain an exponentiation of any order $p$; this is symbolically illustrated in Fig. 4(c).

\section{Square-Rooter Block}

A one-input square rooter has an output with the negative or positive square root of an input voltage multiplied by a constant of a proper polarity, e.g.

$$
V_{0}= \pm\left|\sqrt{K_{R} V_{i}}\right|, \quad K_{R} V_{i}>0 .
$$

Fig. 5(a) shows the implementation of the square rooter, where the output $V_{0}$ is given by

$$
V_{0}=K_{R} \frac{V_{i}}{V_{0}}
$$

which yields

$$
V_{0}= \pm\left|\sqrt{K_{R} V_{i}}\right|
$$

A more detailed description of the implementation is shown in Fig. 5(b). The circuit will be stable if, after a perturbation, the output evolves towards the desired output value. Assume the input is fixed at $V_{i}=U_{s}$. To study the stability and the dynamics of the circuit, a parasitic capacitance $C_{p}$ at the output is again considered. Using the KCL at the output node results in the following nonlinear differential equation:

$$
C_{p} \frac{d V_{0}}{d t}=g_{m} U_{S}+K_{M} V_{0}^{2}
$$

which can be rewritten as

$$
\frac{1}{K_{M}} \frac{d V_{0}}{V_{0}^{2}+\frac{g_{m} U_{S}}{K_{M}}}=\frac{d t}{C_{p}} .
$$

astegrating both sides of (14b) and solving for $V_{0}(t)$ (when $\left.\left(g_{m} / K_{M}\right) U_{S}<0\right)$ yields

$$
V_{0}(t)=K_{i} \frac{1+\frac{V_{0}(0)-K_{i}}{V_{0}(0)+K_{i}} e^{2 K_{i}\left(\frac{K_{M}}{C_{p}}\right) t}}{1-\frac{V_{0}(0)-K_{i}}{V_{0}(0)+K_{i}} e^{2 K_{i}}\left(\frac{K_{M}}{C_{p}}\right) t}
$$

where

$$
K_{i}=\left|\sqrt{\frac{-g_{m} U_{S}}{K_{M}}}\right|
$$

hence

$V_{0}(\infty)=\left\{\begin{aligned} K_{i}=\left|\sqrt{\frac{-g_{m} U_{S}}{K_{M}}}\right|, & \text { for } K_{M}<0 \\ -K_{i}=-\left|\sqrt{\frac{-g_{m} U_{S}}{K_{M}}}\right|, & \text { for } K_{M}>0 .\end{aligned}\right.$

For $\left(g_{m} / K_{M}\right) U_{S}>0$ the solution yields an unbounded output. It is concluded that a stable square-rooter circuit is obtained when $\left(g_{m} / K_{M}\right) V_{i}<0$, i.e., $K_{R} V_{i}>0$. Furthermore, the polarity of $V_{0}$ can be determined according to (15b) or (15c). Fig. 6 shows the conditions for stable operation of the square rooter.

\section{E. Piecewise-Linear Function Generators}

Diodes interconnected with OTA's can simulate ideal diodes, hence allowing the creation of a piecewise-linear approximation to any desired nonlinear function. The ideal basic building blocks for a piecewise-linear function approximation are shown in Fig. 7. High-frequency improve- 


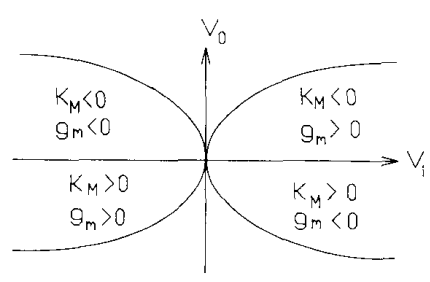

Fig. 6. Stability regions of square rooter.

ments of this basic block are discussed in the Appendix. Note that $I_{D}=0$ until the breaking point (voltage reference $V_{r}$ ) is reached. The slopes of the linear segments are proportional to the $g_{m}$ 's. The diodes can be implemented with MOS transistors with their gate and drain tied together. If a step type input-output characteristic is needed to implement discontinuities in the function approximation, the linear OTA of Fig. 7 can be substituted by an OTA comparator which ideally simulates a large $g_{m}$ and a saturation (output) current of $\pm I_{\mathrm{BIAS}}$.

\section{Nonlinear Function Syntheses}

We present two approaches for nonlinear function syntheses. The first approximation uses a polynomial approach, and the second approximation a piecewise-linear approach.

The first is a rational approximation that has the general form of a polynomial function or of a ratio of polynomials, i.e.,

$$
y_{0}=\frac{\sum_{i=0}^{M} A_{i} x^{i}}{\sum_{i=0}^{N} B_{i} x^{i}}
$$

where $i$ is a positive integer number. In fact, the exponent $i$ can be a fractional exponent of the form $p / q$, where $p$ and $q$ are negative or positive integers. Assume an element $K_{R} x^{p / q}$ needs to be implemented. This is obtained as shown in Fig. 8(a). The exponentiation blocks are of the type of Fig $4(\mathrm{c})$. If a negative $-p / q$ is needed, an additional divider is used as shown in Fig. 8(b).

Next we discuss a piecewise-linear approximation synthesis approach. This approach consists of adding (transconductance) gain segments that have null contribution until a reference (threshold) voltage is reached. A simple but illustrative example is shown in Fig. 9 where a convex curve is approximated. The breakpoints occur at $V_{r_{1}}, V_{r_{2}}$, and $V_{r_{3}}$. The slopes are given by

$$
\begin{array}{lll}
S_{0}=0, & I_{o}=0, & \text { for } V_{r_{1}}>V_{i} \\
S_{1}=-g_{m_{1}}, & I_{o}=-g_{m_{1}} V_{i}, & \text { for } V_{r_{2}}>V_{i}>V \\
S_{2}=-\left(g_{m_{1}}+g_{m_{2}}\right), & I_{o}=-\left(g_{m_{1}}+g_{m_{2}}\right) V_{i}, & \text { for } V_{r_{3}}>V_{i}>V \\
S_{3}=-\left(g_{m_{1}}+g_{m_{2}}+g_{m_{3}}\right), & I_{o}=-\left(g_{m}+g_{m_{2}}+g_{m_{3}}\right) V_{i}, & \text { for } V_{i}>V_{r_{3}} .
\end{array}
$$
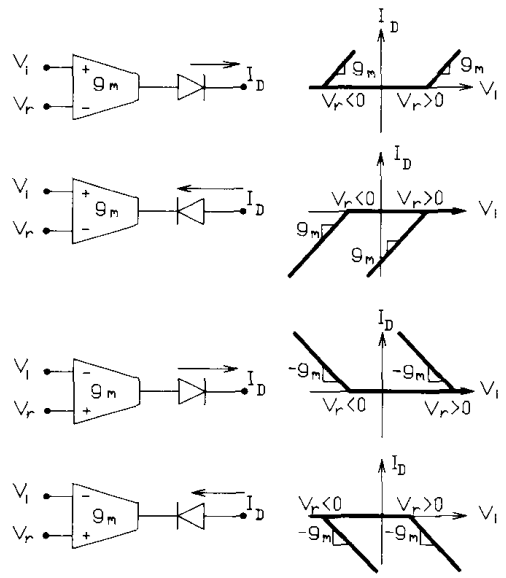

Fig. 7. Piecewise-linear (PL) function generator building blocks.

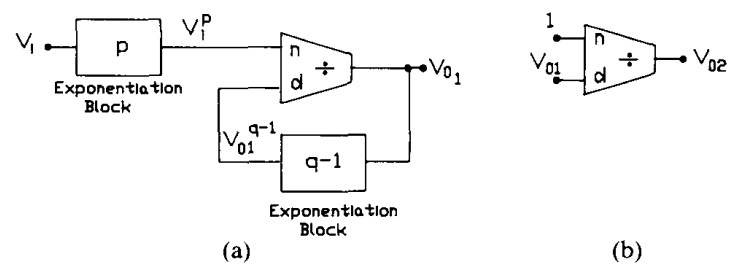

Fig. 8. Fraction power exponentiation: (a) $V_{i}^{p / q}$ and (b) $V_{i}^{-p / q}$.

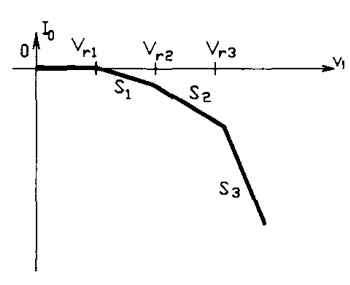

(a)

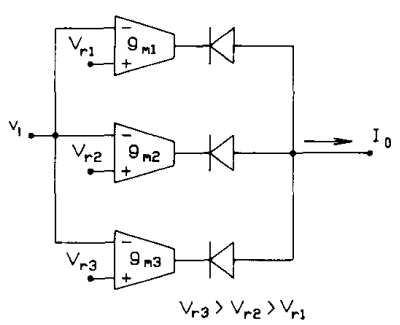

(b)
Fig. 9. Convex curve piecewise-linear approximation: (a) $I_{0}$ versus $V_{i}$ characteristics, and (b) circuit implementation.

Note the continuing increase in magnitude of the slope as the input $v_{i}$ increases, thus forming a convex curve. The approximation will improve as the number of segment lines increases.

Observe that by combining the basic building blocks of Fig. 7, arbitrary functions with variable positive and negative slopes and breakpoints can be approximated. Furthermore, the slopes and breakpoints are voltage programmable, which gives an additional flexibility in the function approximation design problem. Note that if a resistive load simulated with an OTA is used, the slopes become ratios of transconductances which provides a very 


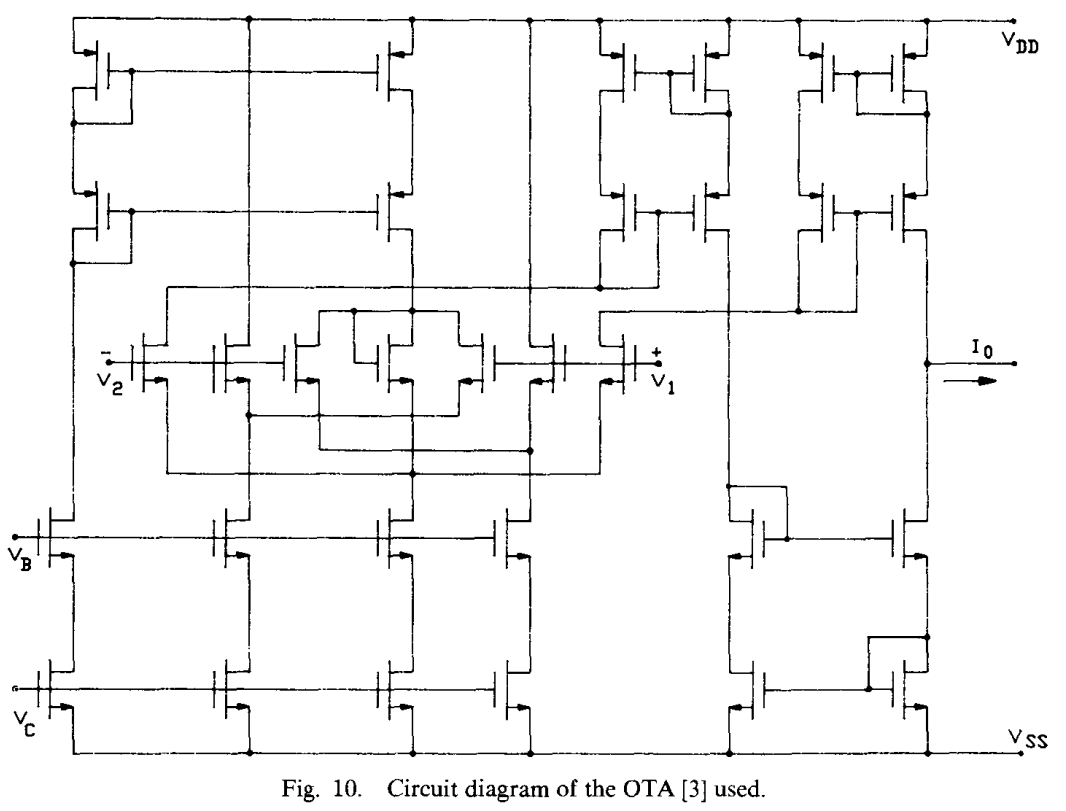

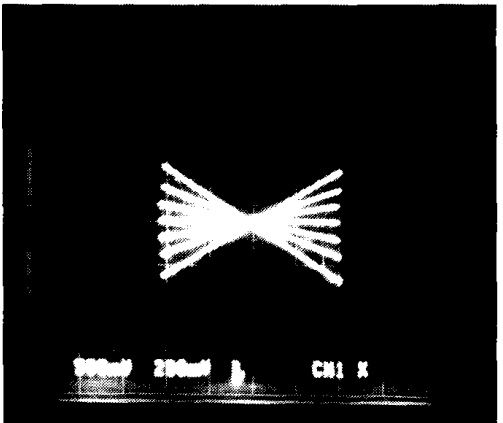

Fig. 11. Large-signal characteristics of multiplier. $V_{1}= \pm\{0.75,0.50$, $0.25,0.0\} \mathrm{V}$.

good temperature compensation [18] and accuracy improvement. In stringent applications where minimum temperature dependence is required, the use of a resistiveload OTA is needed. One example of an arbitrary function approximation containing negative and positive slopes is discussed in the next section. Details on the practical considerations of the OTA-based piecewise-linear circuits are given in the Appendix.

\section{EXPERIMENTAL Results}

Several test circuits containing OTA's and transistors connected as diodes were fabricated using a $3-\mu \mathrm{m}$ p-well CMOS process through (and thanks to) MOSIS. The linearized OTA used to synthesize the different nonlinear analog functions is reported elsewhere [3]. Its schematic is shown in Fig. 10. The OTA has an area of $220 \times 700 \mu \mathrm{m}^{2}$ and consumes $10 \mathrm{~mW}$ for $\pm 5-\mathrm{V}$ supply voltages. In all the examples (unless otherwise indicated) the output current was measured across a $100-\mathrm{k} \Omega$ load resistor.

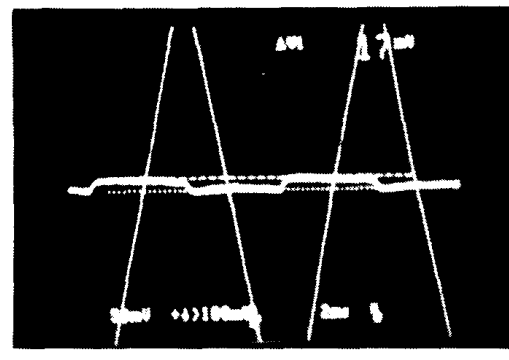

Fig. 12. Nonlinearity multiplier error: fixed $V_{1}=1 \mathrm{~V}$ and variable triangular wave for $V_{2}$

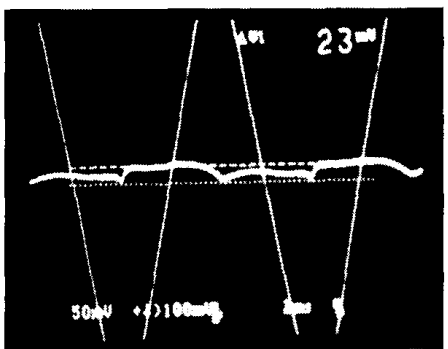

Fig. 13. Nonlinearity multiplier error: fixed $V_{2}=1 \mathrm{~V}$ and variable $V_{1}$

\section{A. Transconductance Multiplier}

The structure used is as shown in Fig. 1. The measured value of $\left|K_{M}\right|$ is $3.3 \mu \mathrm{A} / \mathrm{V}^{2}$. The output current was measured across a $100-\mathrm{k} \Omega$ load resistor. The large-signal characteristics of the multiplier are shown in Fig. 11. $V_{1}$ was held constant (at $0.0, \pm 0.25, \pm 0.50$, and $\pm 0.75 \mathrm{~V}$ ), while the input $V_{2}$ varied between $\pm 1 \mathrm{~V}$. The nonlinearity error is shown in Fig. 12. For $V_{2}$, a triangular 2-V peakto-peak signal was applied, while keeping $V_{1}$ equal to $1 \mathrm{~V}$. 


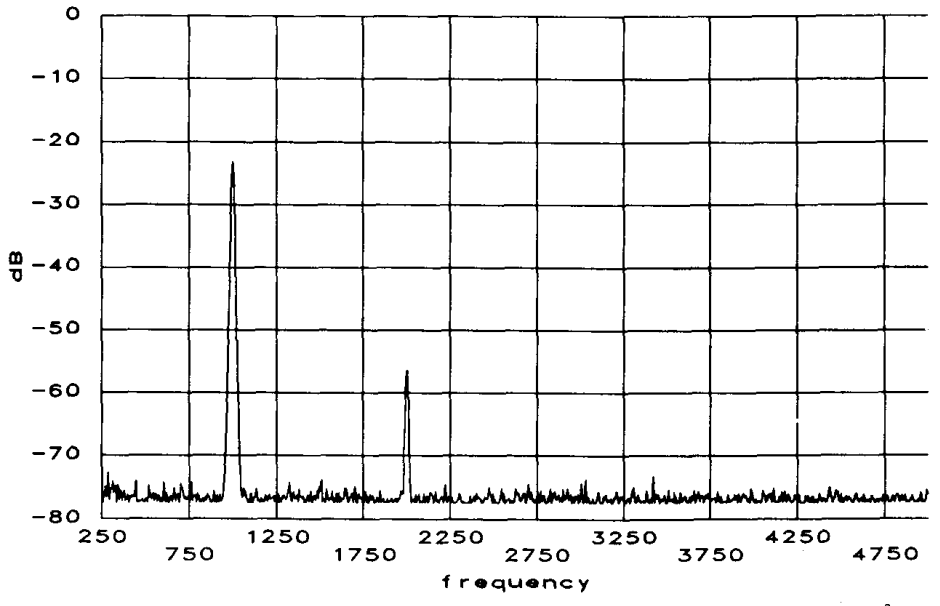

Fig. 14. Spectrum of the multiplier output voltage: $V_{1}=1 \mathrm{~V}$ and $V_{2}=2 \sin \pi \times 10^{3} t$.

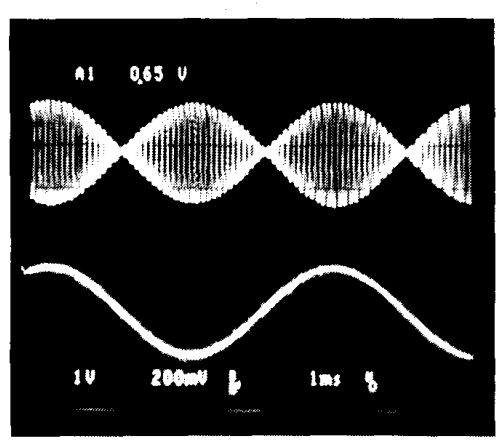

Fig. 15. Modulation for two-input sinusoidal signals.

The output current produced a triangular voltage signal of $660-\mathrm{mV}$ peak to peak. Subtracting this signal from an ideal triangular wave, the resulting peak-to-peak error signal was $17 \mathrm{mV}$, which yields a nonlinearity error of nearly 2 percent. (The ideal triangular wave is a scaled version of the input in such a way that the amplitude of the error signal is minimum.) Repeating the measurement but interchanging $V_{1}$ and $V_{2}\left(V_{1}\right.$ is a triangular signal of $2-\mathrm{V}$ peak to peak), the result obtained is shown in Fig. 13. The peak-to-peak error signal of $23 \mathrm{mV}$ corresponds to a 3.5 percent nonlinearity error. The asymmetry of the multiplier (see inputs in Fig. 1) yields this distortion difference when interchanging the inputs. Making $V_{1}=+1 \mathrm{~V}$ and $V_{2}$ a $2-\mathrm{V}$ peak-to-peak sinusoidal waveform of $1 \mathrm{kHz}$, the spectrum for the multiplier output shown in Fig. 14 was measured. Observe that only the second harmonic, $33 \mathrm{~dB}$ below the fundamental, is present. Fig. 15 shows the multiplier being used as a modulator where both input signals are sinusoidal.

\section{B. Voltage Divider}

The tested circuit has the structure shown in Fig. 2(b) with $\left|K_{M}\right|$ as before in Section IV-A and $K_{M}>0$. The experimental result shown in Fig. 16 was obtained by switching $V_{1}$ between two symmetrical constant values

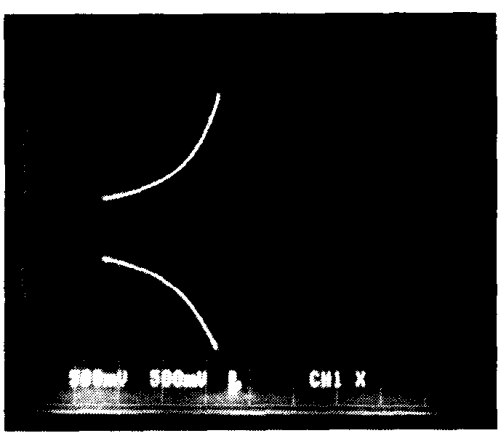

Fig. 16. Divider experimental results: constant $V_{1}$ and varying $V_{2}$.

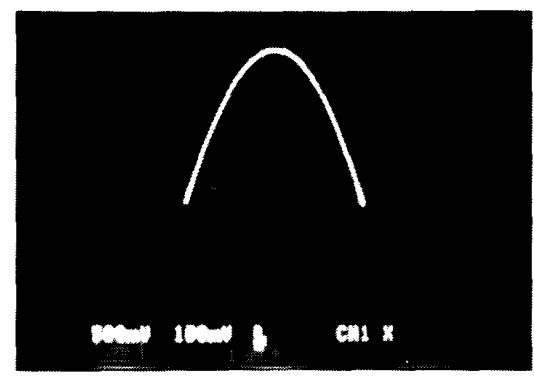

Fig. 17. Squarer experimental results.

( $\pm 1 \mathrm{~V}$ ) while varying $V_{2}\left(V_{2}<0\right)$. This result matches with the theoretical results of Fig. 3(a).

\section{Squarer}

The squarer is obtained by simply making $V_{1}=V_{2}$ in the multiplier discussed in Section IV-A. In this particular case, $K_{M}$ is negative resulting in the inverted parabola shown in Fig. 17. The input range was $\pm 1 \mathrm{~V}$.

\section{Square Rooter}

The basic architecture used is the one shown in Fig. 5(b). The input signal $V_{i}$ is given by $V_{i}=A+A \cos \omega t$ and 


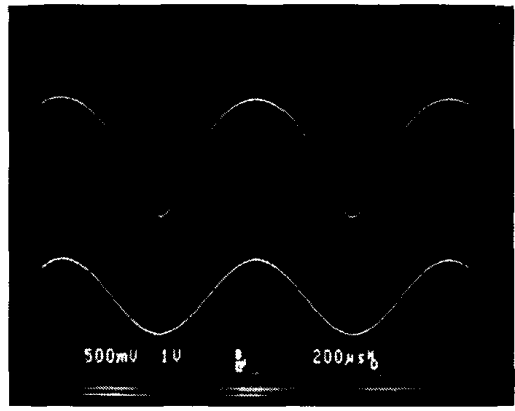

Fig. 18. Square rooter experimental results.

the output, obtained in the first quadrant, has the form

$$
\left|\sqrt{-2 \frac{A g_{m}}{K_{M}}} \cos \frac{\omega}{2} t\right|
$$

where $g_{m}>0, K_{M}<0, A=1 \mathrm{~V}, f=1 \mathrm{kHz}, g_{m}=3.2$ $\mu$ mhos, and $K_{M}=3.3 \mu \mathrm{A} / \mathrm{V}^{2}$. The experimental results are shown in Fig. 18 where the lower trace signal is the input and the output is shown in the upper trace.

\section{E. Piecewise-Linear Approximation}

The intended transfer characteristic is shown in Fig. 19(a) and consists of three linear segments. The individual slopes due to each OTA are indicated in the lower part of Fig. 19(a), and the composed resulting transfer characteristics are shown in the upper part of Fig. 19(a). The actual OTA circuit implementation is shown in Fig. 19(b), where an optional diode and voltage source have been added at the OTA ( 2 and 3 ) to improve the high-frequency performance of the circuit (see the Appendix for more details). Note that the slopes of the transfer characteristics can be easily modified by changing the OTA voltage-dependent transconductances. The experimental results are shown in Fig. 19(c). To show further flexibility, a chip test circuit was reprogrammed to implement a five-segment characteristic to convert a triangular to a sinusoidal waveform. The experimental characteristic is shown in Fig. 30(a), and the input and output waveforms are shown in Fig. 20(b). The measured THD, after optimally adjusting breakpoints and slopes, was 1.5 percent. The chip photomicrograph for these experimental results is shown in Fig. 21.

\section{Conclusions}

The suitability of OTA's as the main active element to obtain basic building blocks for the design of nonlinear networks was established. Methods to implement practical nonlinear circuits in a systematic design approach were developed. Two practical synthesis approaches were introduced. Observe that for both approaches, every time the output current is converted into a voltage by an OTA resistor load, temperature variations are minimized. The programmability and flexibility of the OTA provide the potential to design time-varying nonlinear circuits. The experimental results verified theoretical predictions. Implementations of other nonlinear synthesis approaches [20] are feasible using the basic blocks here introduced. There are many important areas of application of nonlinear functions [22]. One of them is in neural networks [19], [21] as shown by Mead [17, ch. 6]. The proposed OTA-based building blocks can be incorporated in a CAD software [12] to fully exploit their functionality and versatility.

\section{APPENDIX \\ Practical Considerations of the OTA-BASED Piecewise-Linear Circuits}

In this Appendix some practical considerations of the OTA-based piecewise-linear circuits are discussed taking into account some nonidealities of the OTA and of the MOS transistor used as a diode. A simple modification of the circuits of Fig. 7 that leads to a drastic improvement in their high-frequency performance is also presented.

\section{Nonideal Circuit Elements}

Simple equivalent circuits for an MOS OTA and for a diode-connected MOS transistor are shown in Fig. 22. The OTA is characterized by its output impedance (output capacitance $C_{o}$ and output resistance $R_{o}$ ) and input capacitance $\left(C_{i}\right)$. The diode-connected MOS transistor, as shown in Fig. 22(b), is modeled by a resistance ${ }^{4} R_{D}$ in series with a voltage source $V_{t}$ (threshold voltage of the MOS transistor) and an ideal diode, where $\hat{I}_{D}$ is the peak current in the MOS diode. Fig. 22(c) shows the equivalent circuit of the OTA when used as a two-terminal resistive element to simulate a grounded load resistance [6]. The simplified analysis that follows assumes that the transconductance $g_{m}$ of the OTA is frequency independent and neglects the parasitic capacitance of the MOS transistor. ${ }^{5}$ Also, effects due to OTA and diode resistance nonlinearities are not considered.

\section{Low-Frequency Considerations}

The low-frequency equivalent circuit of the OTA precision rectifier of Fig. 23(a) is shown in Fig. 23(b). $g_{m_{2}}$ and $R_{\mathrm{O}_{2}}$ are the transconductance and the output resistance of an OTA used as a load. Parasitic capacitances have been neglected for this low-frequency analysis. Standard circuit

\footnotetext{
${ }^{4}$ In fact, taking into account the mobility degradation [14], $R_{D}$ can be more accurately evaluated, i.e., $R_{D} \cong\left(2 \theta / \mu_{0} C_{o x}\right)(L / W)$.

${ }^{5}$ In most practical cases the time constant $R_{D} C_{D}$ associated with the MOS transistor is negligible compared with other time constants in the circuit.
} 


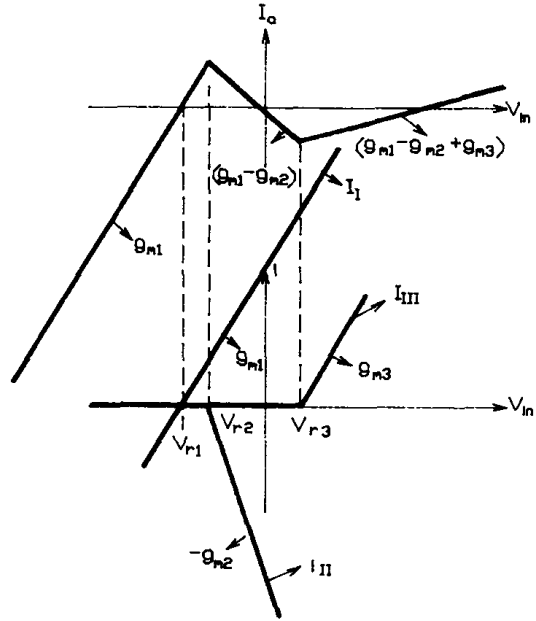

(a)

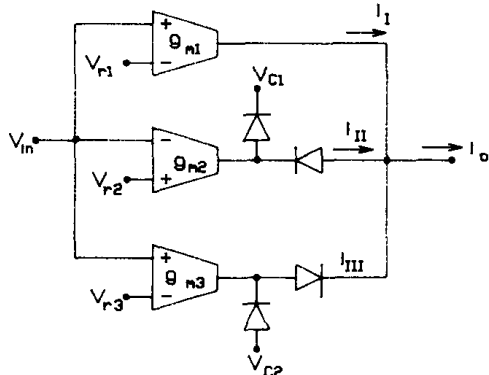

(b)

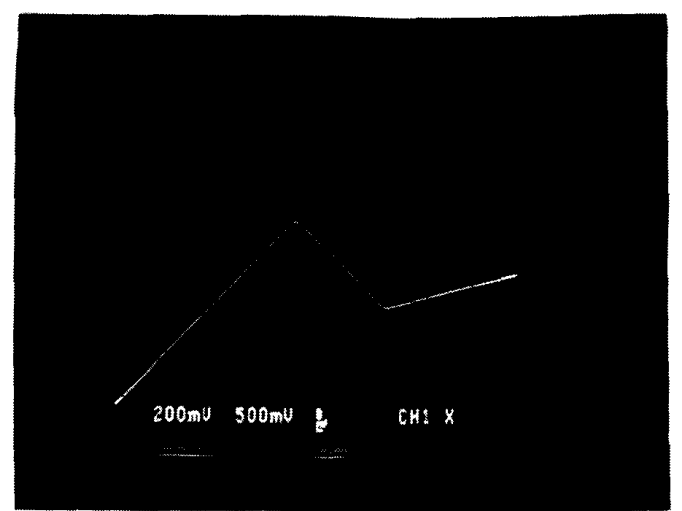

(c)

Fig. 19. Piecewise-linear approximation function: (a) transfer characteristic, (b) circuit implementation, and (c) experimental results.

analysis shows that for $v_{i}>0$ :

$$
v_{0}=\left(g_{m_{1}} R_{o_{1}} v_{i}-V_{t}\right) \cdot \frac{R_{o_{2}} \| \frac{1}{g_{m_{2}}}}{R_{D}+R_{o_{1}}+R_{o_{2}} \| \frac{1}{g_{m_{2}}}} .
$$

Equation (17) can be simplified by assuming $1 / g_{m_{2}} \ll$ $R_{o_{2}}, R_{o_{1}}$. Then

$$
v_{0}=\frac{g_{m_{1}}}{g_{m_{2}}} \cdot \frac{R_{o_{1}}}{R_{D}+R_{o_{1}}} \cdot v_{i}-\frac{V_{t}}{g_{m_{2}}\left(R_{D}+R_{o_{1}}\right)} .
$$

The factor $R_{o_{1}} /\left(R_{D}+R_{o_{1}}\right)$ in the first term of the right side of (18) represents an undesired attenuation. The second term in (18) represents an offset added to the output signal which, added to the offset of the OTA, limits the minimum amplitude of the signal that can be rectified. Assuming $R_{D} \ll R_{o_{1}}$ and $R_{o_{1}} \cong R_{o_{2}}$, an estimated value for the second term is $V_{t} / A_{V_{2}}$ where $A_{V_{2}}=g_{m_{2}} R_{o_{1}}$ is the voltage gain of the OTA 2. Typical values $V_{l} \simeq 1 \mathrm{~V}$ and
$A_{V}=200$ result in approximately $5 \mathrm{mV}$ for this second term. This is also a typical value for the offset voltage of an MOS OTA.

To reduce (18) to the ideal case, i.e.,

$$
v_{0}=\frac{g_{m_{1}}}{g_{m_{2}}} v_{i}, \quad \text { for } v_{i}>0
$$

consider the following practical design considerations for low-frequency applications.

1) Use OTA's with high-voltage gain in order to minimize the offset term in (18) and design the OTA yielding the lowest possible offset voltage. Then limit the minimum input signal amplitude according to (18).

2) Select the $W / L$ ratio of the diode-connected MOS transistor such that $R_{D} \ll R_{o}$ is satisfied. This will make $R_{o_{1}} /\left(R_{o_{1}}+R_{D}\right)=1$. Since the output impedance $R_{o}$ of an OTA is typically very large, this condition in general does not require an excessively large value for the $W / L$ ratio of the MOS transistor. 


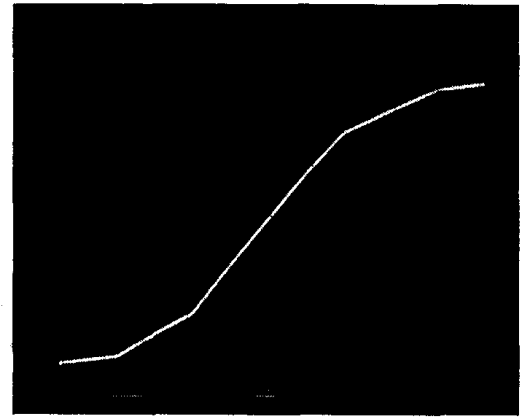

(a)

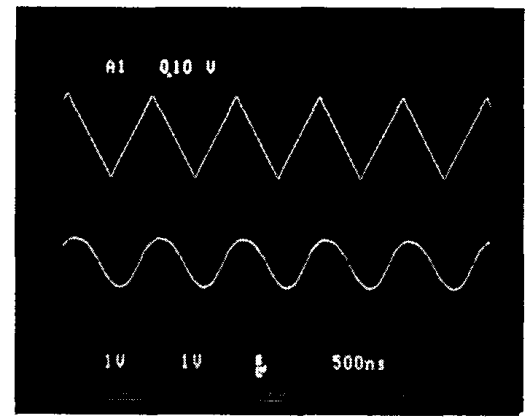

(b)

Fig. 20. (a) Five-segment transfer characteristic. (b) Triangular and sinusoidal waveforms at $1 \mathrm{MHz}$.

\section{High-Frequency Analysis Considerations}

The most important practical factor limiting the highfrequency operation of the circuit of Fig. 23 (see also Fig. 7) is the delay time $t_{D}$ required to discharge the output capacitance $C_{o_{1}}$ from the negative peak voltage $-V_{S}^{-}$ (which is charged during negative half-cycles) to the value $V_{t}$ required for the diode to start conducting. Assuming, for simplicity, that the current during the negative halfcycle is entirely supplied to $C_{o_{1}}$ (with the current in $R_{o}$ considered negligible), the following relationship of the input signal $V_{i}=\hat{V}_{i} \sin \omega t$ involving its frequency $f$ and the delay time $t_{D}$ can be derived from $C_{o_{1}}\left(d V_{C_{o_{1}}} / d t\right)=$ $g_{m} \hat{V}_{i} \sin \omega t$ as ${ }^{6}$ :

$$
f=\frac{I^{\mathrm{MAX}}}{2 \pi C_{o_{1}}\left(V_{t}+\left|V_{S}^{-}\right|\right)}\left[1-\cos \left(2 \pi f t_{D}\right)\right]
$$

where $I^{\operatorname{MAX}}=g_{m} \hat{V}_{i}$ is the peak output current. Fig. 24 illustrates the typical waveform that is observed in the precision rectifier circuit of Fig. 23 for high-frequency operation when $t_{D}$ becomes comparable to the period of

${ }^{6} \mathrm{An}$ additional delay, not considered in the simplified analysis presented here but that can be observed in Fig. 24, is the time taken for the output signal to reach the input signal once the diode is conducting.

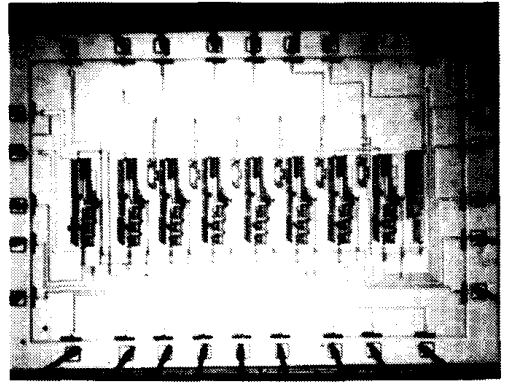

Fig. 21. Microphotograph of chip for piecewise-linear approximation.

the signal, and for the particular case $g_{m_{1}}=g_{m_{2}}$.

If the maximum frequency of operation $f^{\mathrm{MAX}}=1 / T$ is defined arbitrarily as the frequency at which $t_{D}=T / 10$, then from (20) we obtain ${ }^{7}$

$$
f^{\mathrm{MAX}}=\frac{0.19 I^{\mathrm{MAX}}}{2 \pi C_{o_{1}}\left(V_{t}+\left|V_{S}^{-}\right|\right)} .
$$

Typical values of $g_{m}=30 \mu \mathrm{A} / \mathrm{V}, 10 \mathrm{mV} \leqslant \hat{V}_{i} \leqslant 1 \mathrm{~V}, A_{v}=$ $g_{m} R_{o}=100, C_{o_{1}}=0.5 \mathrm{pF}, V_{i}=1 \mathrm{~V}$, and $1 \mathrm{~V} \geqslant\left|V_{S}^{-}\right| \leqslant 5 \mathrm{~V}$ result in a range of $9.07 \mathrm{kHz} \leqslant f^{\mathrm{MAX}} \leqslant 302 \mathrm{kHz}$. From (21) one can see that to increase $f^{\mathrm{MAX}}$ one must increase $I^{\mathrm{MAX}}$ (and hence the power dissipation which is typical for high-frequency circuit performance) and/or design a "better OTA" with smaller $C_{o_{1}}$.

\section{Modification of the Basic Precision Rectifier Circuit to Improve High-Frequency Operation}

Fig. 25 shows high-frequency improved versions of the OTA-rectifier circuits including a second diode ${ }^{8} D_{2}$ and a source $V_{B}=\left(V_{t_{1}}+V_{t_{2}}\right)$ where $V_{t_{1}}$ and $V_{t_{2}}$ are the threshold voltages of $D_{1}$ and $D_{2}$, respectively. In this circuit the OTA1 output voltage does not go into saturation, but is limited to a minimum (maximum) value $V_{t_{1}}\left(-V_{t_{1}}\right)$ during negative (positive) half-cycles. At the beginning of the positive (negative) half-cycles, the diode $D_{1}$ will be ready for conduction so that no delay due to charging or discharging of the output capacitance $C_{o_{1}}$ takes place, hence $t_{D}=0$.

The addition of the diode $D_{2}$ and the bias $V_{B}$ allows a drastic improvement in the high-frequency performance of the circuit without excessively increasing the power consumption. Additional power dissipated still needs to be supplied by the battery $V_{B}$ which absorbs the output current of the OTA through $D_{2}$ during the negative halfcycles, but this does not increase quiescent power dissipation.

The remaining high-frequency limitation of the improved circuits is related to the low-pass behavior of the rectifier. The circuit of Fig. 26 shows a high-frequency equivalent circuit of the OTA rectifier (assumed linear for

${ }^{7}$ Where $\left|V_{S}^{-}\right|=A_{t} \hat{V}_{i}$ for $\left|V_{S}^{-}\right|<V_{S S}$, otherwise $\left|V_{S}^{-}\right| \cong\left|V_{S S}\right|$ and $V_{S S}$ is the negative power supply voltage.

${ }^{8} D_{2}$ works similar to the catch diode of the op-amp version [13] of the same circuit. 


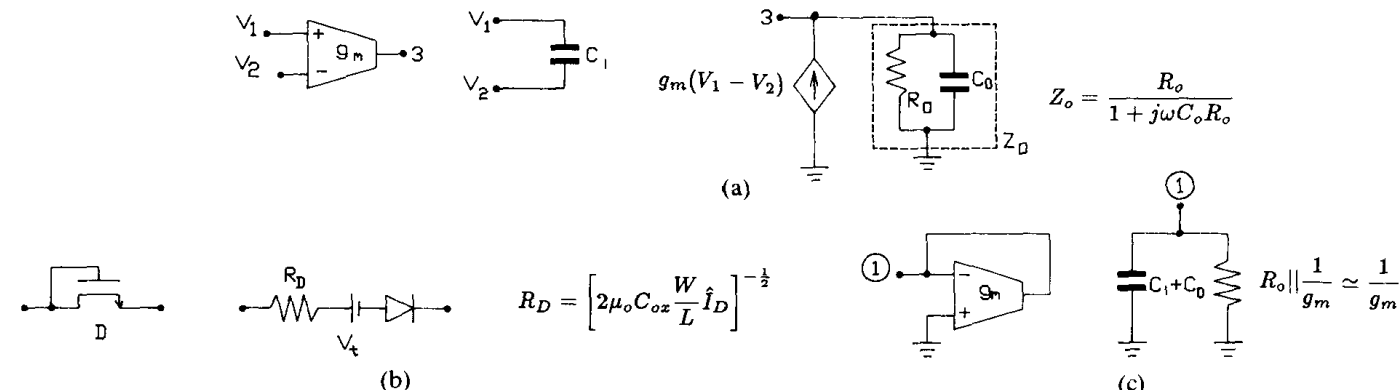

Fig. 22. (a) Equivalent circuit of nonideal OTA. (b) Equivalent circuit of diode-connected MOS transistor. (c) Equivalent circuit of OTA used as two-terminal resistive elements.

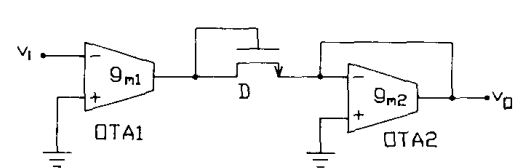

(a)

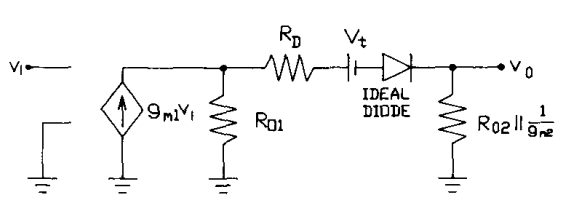

(b)

Fig. 23. (a) Basic OTA-precision rectifier. (b) Low-frequency equivalent circuit of (a).

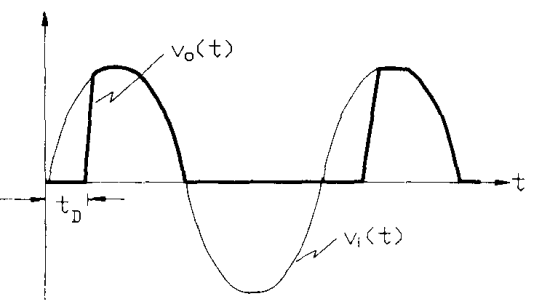

Fig. 24. Nonlinear distortion for high frequency of OTA-precision rectifier of Fig. 23(a).

tractability since the nonlinear transient has been eliminated) for $v_{i}>0$. The transfer function of the circuit, characterized by two poles $\omega_{p_{1}}$ and $\omega_{p_{2}}$, is given by

$$
\frac{v_{o}}{v_{i}}=\frac{g_{m_{1}} / g_{m_{2}}}{\left(1+\frac{s}{\omega_{p_{1}}}\right)\left(1+\frac{s}{\omega_{p_{2}}}\right)}
$$

taking $\omega_{p_{1}}$ and $\omega_{p_{2}}$ into account ${ }^{9}$ and assuming the inequalities, $R_{D},\left(1 / g_{m_{2}}\right) \ll R_{o_{1}}$, are satisfied. The $3-\mathrm{dB}$ frequency, $\omega_{3 \mathrm{~dB}}$, can be approximated [13] by

$$
\omega_{3 \mathrm{~dB}}=\frac{g_{m_{2}}}{C_{o_{1}}\left(1+g_{m_{2}} R_{D}\right)+C_{o_{2}}+C_{i_{2}}} .
$$

If the conditions given above are satisfied, and $1 / g_{m 2} \gg$ $R_{D}$, then we can approximate

$$
\omega_{3 \mathrm{~dB}}=\frac{g_{m_{2}}}{C_{o_{2}}+C_{o_{1}}+C_{i_{2}}} .
$$

For typical values $\left(C_{o_{2}}=C_{o_{1}}=0.5 \mathrm{pF}, C_{i 2}=0.1 \mathrm{pF}, g_{m}=30\right.$ $\mu \mathrm{A} / \mathrm{V}$ ), (24) predicts $f_{3 \mathrm{~dB}}=4.32 \mathrm{MHz}$, which is a factor

$$
\begin{aligned}
& { }^{9} \text { Where } \quad \omega_{p_{1}}=\frac{1}{C_{o_{1}}\left[R_{o_{1}} \|\left(R_{D}+1 / g_{m_{2}}\right)\right]} \text { and } \omega_{p_{2}} \\
& =\frac{1}{\left(C_{o_{2}}+C_{i_{2}}\right)\left[1 / g_{m_{2}} \|\left(R_{D}+R_{o_{1}}\right)\right]} .
\end{aligned}
$$
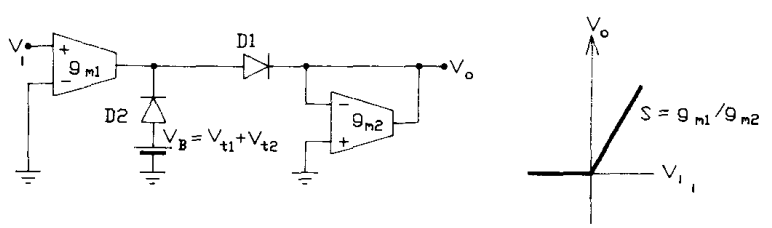

(a)
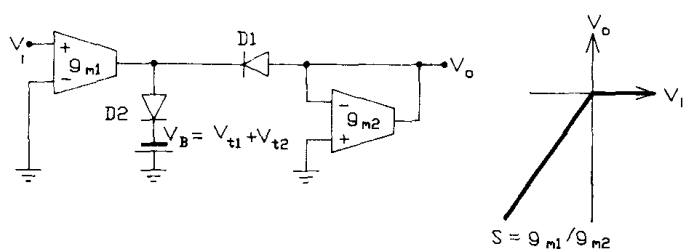

(b)

Fig. 25. Improved versions of OTA-precision rectifiers for highfrequency operation.

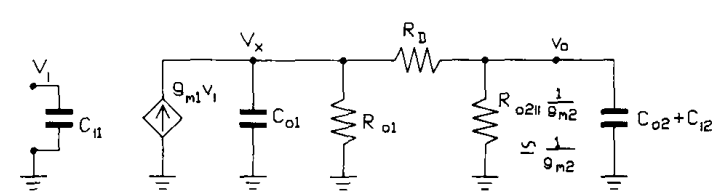

Fig. 26. Small-signal equivalent circuit of improved OTA rectifier of Fig. 25.

between 476 and 14 times higher than the maximum frequency attainable from the basic OTA rectifier. This corresponds to the limit set by the parameters of the OTA used. The conclusion is, then, that the maximum frequency at which OTA precision rectifiers operate can be made as high as the OTA itself allows.

From (23) the following design considerations can be derived to extend $\omega_{3 \mathrm{~dB}}$, which is now effectively the maxi- 
mum frequency of operation of the improved OTA rectifier circuit.

1) Design the OTA with reduced excess phase and parasitic capacitances $C_{o_{1}}, C_{o_{2}}, C_{i_{2}}$, which is, in general, an obvious requirement to improve high-frequency operation.

2) Increase $g_{m_{2}}$ (and also $g_{m_{1}}$ if a fixed value $g_{m_{1}} / g_{m_{2}}$ is desired). Keep $R_{D} \ll 1 / g_{m_{2}}$, that is, assign a sufficiently large $W / L$ ratio for the MOS transistor used as diode so that its resistance is much lower than the load resistance and the multiplying factor of $C_{o_{1}}$ (in (23)) is minimized.

\section{REFERENCES}

[1] R. L. Geiger and E. Sánchez-Sinencio, "Active filter design using operational transconductance amplifiers: A tutorial," IEEE Cir cuits Device Mag., vol. 1, pp. 20-32, Mar. 1985

[2] C. Plett, M. A. Copeland, and R. A. Hadaway, "Continuous-time filters using open-loop tunable transconductance amplifiers," in Proc. ISCAS/IEEE (San Jose, CA), vol. 3, May 1986, pp $1172-1176$.

[3] A. P. Nedungadi and R. L. Geiger, "High-frequency voltage-controlled continuous-time lowpass filters using linearised CMOS intetrolled continuous-time lowpass filters using linearised CMO
grators," Electron. Lett., vol. 22, pp. 729-731, June 1986.

[4] Fr. Krummenacher and N. Joehl, "A 4-MHz CMOS continuous-time filter with on-chip automatic tuning," IEEE J. Solid-State Circuits, vol. 23, pp. $750-758$, June 1988.

[5] E. Sánchez-Sinencio, R. L. Geiger, and H. Nevárez-Lozano, "Generation of continuous-time two integrator loop OTA filter structures," IEEE Trans. Circuits Syst., vol. 35, pp. 936-946, Aug. 1988

[6] M. Bialko and R. W. Newcomb, "Generation of all finite linear circuits using the integrated DVCCS" IEEE Trans. Circuit Theory, vol. CT-18, pp. 733-736, Nov. 1971 .

[7] Linear Databook, National Semiconductor Corp., Santa Clara, CA 1982.

[8] B. Linares-Barranco, A. Rodríguez-Vázquez, J. L. Huertas, E Sánchez-Sinencio, and J. J. Hoyle, "Generation and design of sinusoidal oscillators using OTAs," in Proc. ISCAS/IEEE (Helsinki), vol. 3, June 1988, pp. 2863-2866.

[9] M. A. Chari, K. Nagaraj, and T. R. Viswanathan, "Broad-band precision rectifier," in Proc. ISCAS/IEEE (Philadelphia, PA), precision rectifier, in Proc.
vol. 3, May 1987, pp. $824-826$.

[10] J. W. Fattaruso and R. G. Meyer, "MOS analog function synthesis," IEEE J. Solid-State Circuits, vol. SC-22, pp. 1056-1063, Dec. 1987.

[11] B. S. Song, "CMOS RF circuits for data communication applications," IEEE J. Solid-State Circuits, vol. SC-21, pp. 310-317, Apr. 1986.

[12] L. R. Carley and R. A. Rutenbar, "How to automate analog IC designs," IEEE Spectrum, vol. 25, pp. 26-30, Aug. 1988.

[13] A. S. Sedra and K. C. Smith, Microelectronics Circuits, 2nd ed New York: Holt, Rinehart and Winston, 1987.

[14] C. G. Sodini, P. K. Ko, and J. L. Moll, "The effect of high fields on MOS device and circuit performance," IEEE Trans. Electron Devices, vol. ED-31, pp. 1386-1393, Oct. 1984.

[15] S. Qin and R. L. Geiger, "A $\pm 5-\mathrm{V}$ CMOS analog multiplier," IEEE J. Solid-State Circuits, vol. SC-22, pp. 1143-1146, Dec. 1987

[16] E. Seevinck and R. F. Wassenear, "A versatile CMOS linear transconductor/square-law function circuit," IEEE J. Solid-State Circuits, vol. SC-22, pp. 360-377, June 1987.

[17] C. A. Mead, Analog VLSI and Neural Systems. Reading, MA: Addison-Wesley, 1989.

[18] J. Silva-Martínez and E. Sánchez-Sinencio, "Analogue OTA multiplier without input voltage swing restrictions and temperature compensated," Electron. Lett., vol. 22, pp. 599-600, May 1986.

[19] B. Linares-Barranco, E. Sánchez-Sinencio, A. Rodriguez-Vázquez, and J. L. Huertas, "A programmable neural oscillator cell," IEEE Trans. Circuits Syst. (Special Issue on Neural Networks), vol. 36, pp. 756-761, May 1989

[20] Y. J. Wong and W. E. Ott, Function Circuits, Design and Applications. New York: McGraw-Hill, 1976.

[21] L. O. Chua and L. Yang, "Cellular neural networks: Theory and applications," IEEE Trans. Circuits Syst., vol. 35, pp. 1257-1290, Oct. 1988

[22] D. H. Sheingold, Ed., Nonlinear Circuits Handbook, Analog Devices, Inc., Norwood, MA, 1976

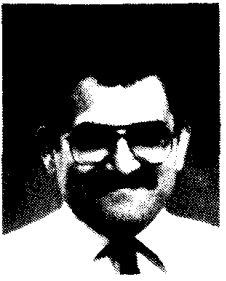

Edgar Sánchez-Sinencio (S'72-M'74-SM'83) was born in Mexico City, Mexico, on October 27, 1944. He received the M.S.E.E. degree from Stanford University, Stanford, CA, in 1970 and the $\mathrm{Ph} . \mathrm{D}$. degree under the tutelage of Professor T. N. Trick from the University of Illinois at Champaign-Urbana in 1973.

In 1974 he held an industrial postdoctoral position with the Central Research Laboratories, Nippon Electric Company, Ltd., Kawasaki, Japan. From 1976 to 1983 , he was the Head of the Department of Electronics at the Instituto Nacional de Astrofísica, Optica y Electrónica, Puebla, Mexico. He was a Visiting Professor in the Department of Electrical Engineering at Texas A\&M University, College Station, during the academic years of 1979-1980 and 1983-1984, where he is currently a Professor. He is the co-author of the book SwitchedCapacitor Circuits (Van Nostrand-Reinhold, 1984). His present interests are in the area of solid-state circuits, including CMOS neural network implementations, and computer-aided circuit design.

Dr. Sánchez-Sinencio was the General Chairman of the 1983 26th Midwest Symposium on Circuits and Systems. He was an Associate Editor of News and Events for the IEEE Circuits and Devices Magazine from 1985 to 1988 . He was an Associate Editor for the IEEE TRANSACTIONS ON CIRCUITS AND SYSTEMS from 1985 to 1987 . He is currently the President-Elect of the IEEE Circuits and Systems Technical Committee on Neural Systems and Applications.

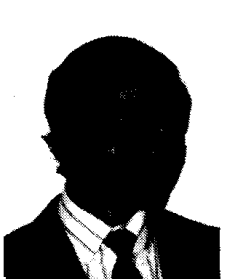

Jaime Ramírez-Angulo (M'76) received the B.S.C and M.Sc. Eng. degrees in electrical engineering from the National Polytechnic Institute, Mexico, in 1973 and 1976, respectively, and the Ph.D. degree in electrical engineering from the University of Stuttgart, West Germany, in 1982.

He worked from 1982 to 1984 at the National Institute for Astrophysics, Optics and Electronics (INAOE) in Mexico. Since 1984 he has been an Assistant Professor in the Electrical Engineering Department of Texas A\&M University, College Station. His research interests are in the areas of analog filters, analog microelectronic circuit design, and CAD for analog systems.

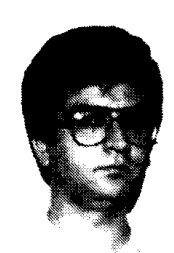

Bernabé Linares-Barranco was born in Granada Spain, on November 26, 1962 . He did his elementary studies in Germany until 1973. He received the B.Sc. degree in electronic physics in June 1986, and the M.S. degree in microelectronics in September 1987, both from the University of Seville, Spain.

Currently, he is with the Department of Electrical Engineering, Texas A\&M University, College Station, where he is working towards the $\mathrm{Ph} . \mathrm{D}$. degree. His research interests are in the area of nonlinear analog microelectronic design and neural networks.

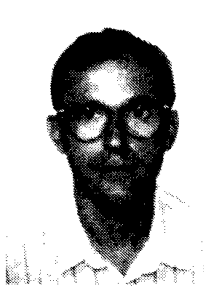

Angel Rodríguez-Vázquez (M'80) was born in Seville, Spain. He received the Licenciado en Fisica degree in 1977, and the Doctor en Ciencias Físicas degree in 1983, both from the University of Seville, Spain.

Since October 1978 he has been with the Departamento de Electrónica y Electromagnetismo at the University of Seville, where he is currently an Associate Professor. His research interest lies in the fields of analog/digital integrated circuit design, analog integrated neural and nonlinear networks, and modeling of analog integrated circuits. 